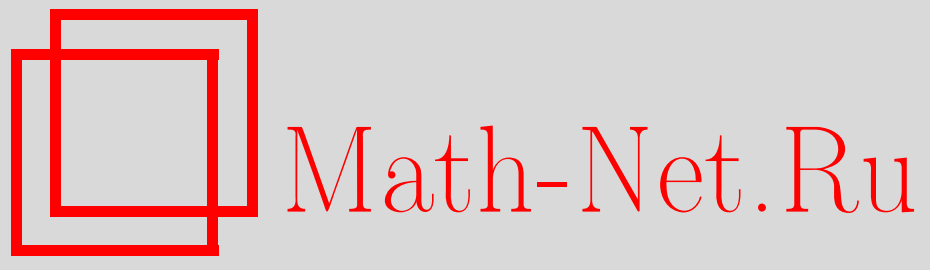

А. В. Сюлюкин, Об асимптотических разложениях для сверток распределений, принадлежащих зонам притяжения устойчивых законов, Теория вероятн. и ее примен., 2013, том 58, выпуск 3, 608-614

DOI: https://doi.org/10.4213/tvp4531

Использование Общероссийского математического портала Math-Net.Ru подразумевает, что вы прочитали и согласны с пользовательским соглашением http://www . mathnet.ru/rus/agreement

Параметры загрузки:

IP: 18.208 .226 .222

26 апреля 2023 г., 15:40:01

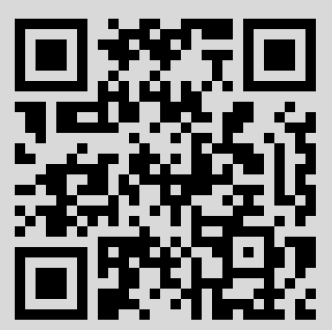


(c) 2013 г.

СЮЛЮКИН А.В.*

\title{
ОБ АСИМПТОТИЧЕСКИХ РАЗЛОЖЕНИЯХ ДЛЯ СВЕРТОК РАСПРЕДЕЛЕНИЙ, ПРИНАДЛЕЖАЩИХ ЗОНАМ ПРИТЯЖЕНИЯ УСТОЙЧИВЫХ ЗАКОНОВ
}

\begin{abstract}
Рассмотрены асимптотические разложения, уточняющие предельные теоремы с предельными устойчивыми распределениями. Получены явные оценки остаточных частей таких разложений.
\end{abstract}

Ключевые слова и фразы: предельные теоремы в схеме накопленных сумм, асимптотические разложения, точность аппроксимации.

Хорошо известна задача об асимптотических разложениях, уточняющих предельные теоремы с устойчивыми предельными законами. Одни из первых результатов здесь принадлежат Г. Крамеру (см. [5, гл. IV, §7, п. 2, с. 239]). Дальнейшее развитие эта задача получила в работах [1], [10]-[12] и др.

В цитированных статьях установлены асимптотические разложения для нормированных сверток распределений с оценками остаточных частей, выписанных в терминах о-символики. Явные оценки остаточных частей не приводятся, между тем такие оценки известны для разложений Эджворта (и их модификаций), уточняющих центральную предельную теорему (см. [7]). Цель настоящей статьи - выписать несколько асимптотических разложений для случая устойчивых предельных распределений с явными оценками их остаточных частей. Приведенные разложения являются частными случаями разложений из [10] и аналогами разложения из [8], вместе с тем они достаточно просты и с их помощью можно моделировать распределения (в том числе $n$-мерные), близкие к устойчивым законам. Отметим, что приемы, предложенные в работе, могут быть использованы и для оценки остаточных частей более общих разложений из [10] и [11].

1. Одномерные разложения. Введем обозначения: $P(x)$ - функция распределения, соответствующая вероятностной мере $P ; S(x)$ - функция распределения некоторого устойчивого закона; $s(x)$ - плотность $S(x) ; \phi_{S}(t)$ - характеристическая функция $S ; 0<\alpha<2, d>0, \alpha, d$ - числа, определяемые из соотношения $\left|\phi_{S}(t)\right|=\mathrm{e}^{-d|t|^{\alpha}}$; $S_{n-l}(x)=S\left((n /(n-l))^{1 / \alpha} x\right) ; s_{n-l}(x)$ - плотность $S_{n-l}(x) ; n, l, n-l \in \mathbf{N}$;

$$
\begin{gathered}
\Delta_{j}=\int_{-\infty}^{\infty} x^{j} d(P-S)(x), \quad|\Delta|_{j}=\int_{-\infty}^{\infty}|x|^{j}|d(P-S)(x)|, \quad j \in \mathbf{Z}_{+} ; \\
B_{k}^{j}(d)=\frac{1}{2 \pi} \int_{-\infty}^{\infty}|t|^{j} \mathrm{e}^{-d(k / n)|t|^{\alpha}} d t, \quad \text { где } j \in \mathbf{Z}_{+}, \quad k, n \in \mathbf{N}, \quad d \in \mathbf{R} ; \\
\alpha(T)=\max _{t \geqslant T} \max \left\{|f(t)|, \mathrm{e}^{-d|t|^{\alpha}}\right\} .
\end{gathered}
$$

Везде далее, где будут использованы величины $\Delta_{j},|\Delta|_{j}$, предполагается, что они конечны. Отметим, что $|\Delta|_{j}$ - вариация с весом $|x|^{j}$ между распределениями $P$ и $S$. Будем называть $|\Delta|_{j}$ псевдомоментом порядка $j$ распределения $P$.

Нам понадобится следующее условие на характеристическую функцию $f(t)$ распределения $P$ :

$$
\int_{-\infty}^{\infty}|f(t)|^{\nu} d t<\infty \quad \text { для некоторого } \nu>0 \text {. }
$$

* Институт проблем безопасного развития атомной энергетики РАН, Москва, Россия; e-mail: syulyukin@yandex.ru 
Теорема 1. Пусть $\alpha \in(0,1)$, абсолютный псевдомомент порядка $m+1, m \in \mathbf{N}$, распределения $P$ конечен и выполнено условие (1). Тогда для $n>\nu+M$, где $M=$ $M(m)=[m /(1-\alpha)]+2$, существует плотность $p_{n}(x)$ распределения $P^{* n}\left(n^{1 / \alpha} A\right)$, $A \in \mathfrak{B}(\mathbf{R})$, такая, ито

$$
p_{n}(x)=s(x)+\sum_{l=1}^{M-1} C_{n}^{l} \sum_{k=0}^{m-1} \frac{(-1)^{l+k} s_{n-l}^{(l+k)}(x)}{n^{(l+k) / \alpha}} \sum_{\substack{i_{1}, \ldots, i_{l} \geqslant 1 \\ i_{1}+\cdots+i_{l}=l+k}} \frac{\Delta_{i_{1}} \cdots \Delta_{i_{l}}}{i_{1} ! \cdots i_{l} !}+R_{m+1},
$$

где для любого $x \in \mathbf{R}$

$$
\begin{gathered}
\left|R_{m+1}\right| \leqslant \sum_{l=1}^{M-1} C_{n}^{l} \frac{B_{n-l}^{l+m}(d)}{n^{(l+m) / \alpha}} \sum_{\substack{i_{1}, \ldots, i_{l} \geqslant 1 \\
i_{1}+\cdots+i_{l}=l+m}} \frac{|\Delta|_{i_{1}} \cdots|\Delta|_{i_{l}}}{i_{1} ! \cdots i_{l} !}+C_{n}^{M} \frac{|\Delta|_{1}^{M} B_{n-M}^{M}(d / 2)}{n^{M / \alpha}} \\
+\frac{2^{M}}{\pi} n^{1 / \alpha} C_{n}^{M} \alpha^{n-\nu-M}(T) \int_{T}^{\infty} \max ^{\nu}\left\{|f(t)|, \mathrm{e}^{-d|t|^{\alpha}}\right\} d t, \\
T=\min \left\{\left(\frac{1}{4|\Delta|_{1}}\right)^{1 /(1-\alpha)},\left(\frac{1}{2 d}\right)^{1 / \alpha}\right\} .
\end{gathered}
$$

3 а м е ч а н и е 1 . Число $M=M(m)$ выбрано наименьшим целым числом, для которого

$$
C_{n}^{M} \frac{|\Delta|_{2}^{M} B_{n-M}^{M}(d / 2)}{(2 !)^{M} n^{M / \alpha}}=O\left(\frac{1}{n^{(m+1) / \alpha-1}}\right), \quad R_{m+1}=O\left(\frac{1}{n^{(m+1) / \alpha-1}}\right) .
$$

Индекс $m+1$ у величины $R_{m+1}$ указывает на то, что в оценке остаточной части используются абсолютные псевдомоменты вплоть до порядка $m+1$.

Заметим, что производные любых порядков плотностей устойчивых распределений существуют. Явные выражения для них в общем случае неизвестны, но для производных плотностей устойчивых законов можно получать представления в виде асимптотических рядов. Асимптотические ряды для самих плотностей можно найти, например, в [2], [3].

Теорема 2. Пусть $\alpha \in[1,2)$, первый псевдомомент распределения $P$ равен нулю, абсолютньй псевдомомент порядка $m+2, m \in \mathbf{N}$, конечен и выполнено условие (1). Тогда для $n>\nu+M$, где

$$
M=M(m)= \begin{cases}{\left[\frac{m}{2-\alpha}\right]+2,} & \alpha \in(1,2), \\ m+1, & \alpha=1,\end{cases}
$$

существует плотность $p_{n}(x)$ распределения $P^{* n}\left(n^{1 / \alpha} A\right), A \in \mathfrak{B}(\mathbf{R})$, такая, ито

$$
p_{n}(x)=s(x)+\sum_{l=1}^{M-1} C_{n}^{l} \sum_{k=0}^{m-1} \frac{(-1)^{2 l+k} s_{n-l}^{(2 l+k)}(x)}{n^{(2 l+k) / \alpha}} \sum_{\substack{i_{1}, \ldots, i_{l} \geqslant 2 \\ i_{1}+\cdots+i_{l}=2 l+k}} \frac{\Delta_{i_{1}} \cdots \Delta_{i_{l}}}{i_{1} ! \cdots i_{l} !}+R_{m+2},
$$

где для любого $x \in \mathbf{R}$

$$
\begin{gathered}
\left|R_{m+2}\right| \leqslant \sum_{l=1}^{M-1} C_{n}^{l} \frac{B_{n-l}^{2 l+m}(d)}{n^{(2 l+m) / \alpha}} \sum_{\substack{i_{1}, \ldots, i_{l} \geqslant 2 \\
i_{1}+\cdots+i_{l}=2 l+m}} \frac{|\Delta|_{i_{1}} \cdots|\Delta|_{i_{l}}}{i_{1} ! \cdots i_{l} !}+C_{n}^{M} \frac{\left.|\Delta|\right|_{2} ^{M} B_{n-M}^{2 M}(d / 2)}{(2 !)^{M} n^{2 M / \alpha}} \\
+\frac{2^{M}}{\pi} n^{1 / \alpha} C_{n}^{M} \alpha^{n-\nu-M}(T) \int_{T}^{\infty} \max ^{\nu}\left\{|f(t)|, \mathrm{e}^{-d|t|^{\alpha}}\right\} d t \\
T=\min \left(\left(\frac{1}{2|\Delta|_{2}}\right)^{1 /(2-\alpha)},\left(\frac{1}{2 d}\right)^{1 / \alpha}\right) .
\end{gathered}
$$


3 а м е ч а н и е 2 . Число $M=M(m)$ выбрано наименьшим целым числом, для которого

$$
C_{n}^{M} \frac{|\Delta|_{2}^{M} B_{n-M}^{2 M}(d / 2)}{(2 !)^{M} n^{2 M / \alpha}}=O\left(\frac{1}{n^{(m+2) \alpha-1}}\right), \quad R_{m+2}=O\left(\frac{1}{n^{(m+2) / \alpha-1}}\right) .
$$

Из утверждения о конечности некоторого абсолютного псевдомомента распределения $P$ следует, что разность характеристических функций распределений $P$ и $S$ разлагается в нуле в отрезок ряда Тейлора. Приведем асимптотическое разложение с явной оценкой в случае, когда отношение характеристических функций разложимо в ряд Тейлора.

Теорема 3. Если

$$
f(t)=\phi_{S}(t)\left(1+\alpha_{1} i t+\cdots+\alpha_{m+1} \frac{(i t)^{m+1}}{(m+1) !}+\beta_{m+2} \frac{(i t)^{m+2}}{(m+2) !} \gamma(t)\right),
$$

где $\gamma(t)-$ комплекснозначная функция, $|\gamma(t)| \leqslant 1, \alpha_{i} \in \mathbf{R}, 1 \leqslant i \leqslant m+1, \beta_{m+2}>0$, $m \in \mathbf{N}$, то для плотности $p_{n}(x)$ верно разложение из теоремы 1 с заменой, для всех встречаюоихся в этом разложении $j$, величин $s_{n-l}^{(j)}(x), \Delta_{j}$ из главной части на $s^{(j)}(x), \alpha_{j}$, а величин

$$
B_{n-l}^{j}(d), \quad B_{n-M}^{M}\left(\frac{d}{2}\right), \quad|\Delta|_{j}, \quad T=\min \left\{\left(\frac{1}{4|\Delta|_{1}}\right)^{1 /(1-\alpha)},\left(\frac{1}{2 d}\right)^{1 / \alpha}\right\}
$$

на

$$
B_{n}^{j}(d), \quad B_{n}^{M}\left(\frac{d}{2}\right), \quad \beta_{j}, \quad T=\left(\frac{d}{2|\Delta|_{1}}\right)^{1 /(1-\alpha)}
$$

из оченки остаточной части.

3 а м е ч а н и е 3 . Для характеристической функции $f(t)$ из теоремы 3 условие (1) выполнено при любом $\nu>0$.

Далее для краткости мы будем формулировать утверждения для случая $\alpha \in[1,2)$.

Аналогом теоремы 2 для функций распределения является следующее утверждение.

Теорема 4. Пусть $\alpha \in[1,2)$, первый псевдомомент между распределениями $P$ $u S$ равен нулю, абсолютный псевдомомент порядка $m+2, m \in \mathbf{N}$, конечен. Тогда для функиии распределения $P_{n}(x)$ меры $P^{* n}\left(n^{1 / \alpha} A\right), A \in \mathfrak{B}(\mathbf{R})$, при $n>\nu+M$, где $M$ определено в (2), верно разложение

$$
P_{n}(x)=S(x)+\sum_{l=1}^{M-1} C_{n}^{l} \sum_{k=0}^{m-1} \frac{(-1)^{2 l+k} S_{n-l}^{(2 l+k)}(x)}{n^{(2 l+k) / \alpha}} \sum_{\substack{i_{1}, \ldots, i_{l} \geqslant 2 \\ i_{1}+\cdots+i_{l}=2 l+k}} \frac{\Delta_{i_{1}} \cdots \Delta_{i_{l}}}{i_{1} ! \cdots i_{l} !}+R_{m+2},
$$

где для любого $x \in \mathbf{R}$

$$
\begin{aligned}
\left|R_{m+2}\right| \leqslant & \sum_{l=1}^{M-1} C_{n}^{l} \frac{B_{n-l}^{2 l+m-1}(d)}{n^{(2 l+m) / \alpha}} \sum_{\substack{i_{1}, \ldots, i_{l} \geqslant 2 \\
i_{1}+\cdots+i_{l}=2 l+m}} \frac{|\Delta|_{i_{1}} \cdots|\Delta|_{i_{l}}}{i_{1} ! \cdots i_{l} !} \\
& +C_{n}^{M} \frac{|\Delta|_{2}^{M} B_{n-M}^{2 M-1}(d / 2)}{(2 !)^{M} n^{2 M / \alpha}}+\frac{24 \eta_{M, n}}{\pi T^{n} n^{n / \alpha}} \\
& +\frac{2^{M+1}(n-1)}{\pi} C_{n}^{M} \theta^{n-M} \max \left\{0, \ln \left(T n^{1 / \alpha}\right)\right\}, \\
\eta_{M, n}= & \sum_{j=0}^{M-1} \frac{C_{n}^{j} B_{n-j}^{2 j}|\Delta|_{2}^{j}}{2^{j} n^{2 j / \alpha}}, \quad \theta=\sup _{|t| \geqslant T}|f(t)|,
\end{aligned}
$$




$$
T=\min \left\{\left(\frac{1}{2|\Delta|_{2}}\right)^{1 /(2-\alpha)},\left(\frac{1}{2 d}\right)^{1 / \alpha}\right\} .
$$

3 а м е ч а н и е 4. Если характеристическая функция $f(t)$ распределения $P$ такова, что $\varlimsup_{|t| \rightarrow \infty}|f(t)|<1$, то $\theta<1$; при этом $\eta_{M, n} \rightarrow(2 \pi)^{-1} \int_{-\infty}^{\infty} \mathrm{e}^{-d|t|^{\alpha}} d t, n \rightarrow \infty$.

2. Многомерное разложение. Пусть $x \in \mathbf{R}^{D}$, и пусть характеристическая функция $\phi_{S}(t), t \in \mathbf{R}^{D}$, устойчивого распределения $S(x)=S(\alpha, \Lambda, \mu, x), 0<\alpha<2$, имеет вид

$$
\phi_{S}(t)=\exp \left(i t^{T} \mu-\int_{\mathbf{S}}\left(\left|t^{T} u\right|^{\alpha}+i \nu\left(t^{T} u, \alpha\right)\right) \Lambda(d u)\right),
$$

где

$$
\nu(y, \alpha)= \begin{cases}-\operatorname{sign}(y) \operatorname{tg}\left(\frac{\pi \alpha}{2}\right)|y|^{\alpha}, & \alpha \neq 1, y \in \mathbf{R}, \\ \frac{2}{\pi} y \ln |y|, & \alpha=1,\end{cases}
$$

$\Lambda$ - конечная мера на единичной сфере $\mathbf{S}=\left\{u \in \mathbf{R}^{D}:|u|=1\right\}, \mu \in \mathbf{R}^{D}$. Для $x \in \mathbf{R}^{D}$ введем следующие обозначения: $P(x)$ - функция распределения, соответствующая вероятностной мере $P ; S(x)$ - функция распределения некоторого устойчивого закона; $s(x)$ - плотность $S(x) ; S_{n-l}(x)=S\left((n /(n-l))^{1 / \alpha} x\right) ; s_{n-l}(x)$ - плотность $S_{n-l}(x) ; n, l, n-l \in \mathbf{N} ; s_{n-l}^{(l)}(x)[h]-l$-я производная по Фреше, взятая в вектор-точке $x$ и примененная к вектору $h$ (подробнее см. [4], [6]); $Q=P-S$;

$$
\begin{gathered}
|\Delta|_{j}=\sup _{|e|=1, e \in \mathbf{R}^{D}} \int_{\mathbf{R}^{D}}|(x, e)|^{j}|d(P-S)(x)|, \quad j \in \mathbf{Z}_{+} ; \\
\alpha(T)=\max _{|t| \geqslant T} \max \left\{|f(t)|, \mathrm{e}^{-d|t|^{\alpha}}\right\} ; \\
\Delta_{1}(e)=\int_{\mathbf{R}^{D}}(x, e) d(P-S)(x) ; \quad B_{k}^{j}(d)=\frac{1}{(2 \pi)^{D}} \int_{\mathbf{R}^{D}}|t|^{j} \mathrm{e}^{-d(k / n)|t|^{\alpha}} d t,
\end{gathered}
$$

где $j \in \mathbf{Z}_{+}, k, n \in \mathbf{N}, d \in \mathbf{R}$. Обобщение условия (1) на $D$-мерный случай имеет вид

$$
\int_{\mathbf{R}^{D}}|f(t)|^{\nu} d t<\infty \quad \text { для некоторого } \nu>0 .
$$

Теорема 5. Пусть $\Delta_{1}(e)=0$ для любого е $\in \mathbf{R}^{D}$, и пусть выполнень остальные $D$-мернье аналоги условий теоремь 2. Тогда при $n>\nu+M$, где $M$ определено в (2), справедливо разложение

$$
\begin{aligned}
p_{n}(x)= & s(x)+\sum_{l=1}^{M-1} C_{n}^{l} \sum_{k=0}^{m-1}(-1)^{2 l+m} \\
& \times \int_{\mathbf{R}^{D}} \cdots \int_{\mathbf{R}^{D}} \frac{s_{n-l}^{(2 l+k)}(x)\left[h_{1}+\cdots+h_{l}\right]}{n^{(2 l+k) / \alpha}(2 l+k) !} Q\left(d h_{1}\right) \cdots Q\left(d h_{l}\right)+R_{m+2},
\end{aligned}
$$

где для любого $x \in \mathbf{R}^{D}$

$$
\begin{aligned}
&\left|R_{m+2}\right| \leqslant \sum_{l=1}^{M-1} C_{n}^{l} \frac{B_{n-l}^{2 l+m}(d)}{n^{(2 l+m) / \alpha}} \sum_{\substack{i_{1}, \ldots, i_{l} \geqslant 2 \\
i_{1}+\cdots+i_{l}=2 l+m}} \frac{|\Delta|_{i_{1}} \cdots|\Delta|_{i_{l}}}{i_{1} ! \cdots i_{l} !}+C_{n}^{M} \frac{|\Delta|_{2}^{M} B_{n-M}^{2 M}(d / 2)}{(2 !)^{M} n^{2 M / \alpha}} \\
&+ \frac{2^{M}}{(2 \pi)^{D}} n^{D / \alpha} C_{n}^{M} \alpha^{n-\nu-M}(T) \int_{|t| \geqslant T} \max ^{\nu}\left\{|f(t)|, \mathrm{e}^{-d|t|^{\alpha}}\right\} d t, \\
& T=\min \left\{\left(\frac{1}{2|\Delta|_{2}}\right)^{1 /(2-\alpha)},\left(\frac{1}{2 d}\right)^{1 / \alpha}\right\}, \quad d=\Lambda(\mathbf{S}) .
\end{aligned}
$$


Рассмотрим двумерное распределение Коши с плотностью $\kappa(x, y)=(2 \pi)^{-1}(1+$ $\left.x^{2}+y^{2}\right)^{-3 / 2}$. В условиях теоремы 5 с предельным распределением $\kappa(x, y)$ и $m=1$ имеем:

$$
\begin{aligned}
& p_{n}(x, y)=\kappa(x, y)+\left(\frac{n}{n-1}\right)^{2} \frac{\kappa_{1}^{-5 / 2}(x, y)}{2 \pi n} \\
& \quad \times\left(\frac{15}{2} \frac{n}{n-1} \kappa_{1}^{-1}(x, y)\left(\Delta_{2, x} x^{2}+2 \Delta_{2, x y} x y+\Delta_{2, y} y^{2}\right)-3 \Delta_{2, x}-3 \Delta_{2, y}\right)+O\left(\frac{1}{n^{2}}\right),
\end{aligned}
$$

где

$$
\begin{gathered}
\Delta_{2, x}=\int_{\mathbf{R}^{2}} x^{2} d Q(x, y), \quad \Delta_{2, x y}=\int_{\mathbf{R}^{2}} x y d Q(x, y), \\
\kappa_{1}(x, y)=\left\{1+\left(\frac{n}{n-1} x\right)^{2}+\left(\frac{n}{n-1} y\right)^{2}\right\}^{-1} .
\end{gathered}
$$

3. Об оптимизации разложений. Известны так называемые короткие, средние и длинные асимптотические разложения в центральной предельной теореме (см. [7]). Аналогичные утверждения можно сформулировать и в случае сходимости к произвольному устойчивому закону. Например, в следующем утверждении приводится некоторый набор асимптотических разложений. Каждое разложение получается с помощью выбора индексов суммирования $N(l)$ (см. далее).

Теорема 6 (о выборе оптимального разложения). Пусть выполнень условия теоремь 2 с $\alpha=1$. Пусть $N(l)$ - произвольные числа, удовлетворяюшие неравенствам $l+1 \leqslant N(l) \leqslant 2 l, 1 \leqslant l \leqslant m$. Тогда при $n>m+\nu+1$ справедливо разложение

$$
\begin{aligned}
p_{n}(x)= & s(x)+\sum_{l=1}^{m} C_{n}^{l} \sum_{\substack{k=0 \\
m+N(l)-2 l-1}} \frac{(-1)^{2 l+k} s_{n-l}^{(2 l+k)}(x)}{n^{2 l+k}} \\
& \times \sum_{\substack{i_{1}, \ldots, i_{l} \geqslant 2 \\
i_{1}+\cdots+i_{l}=2 l+k}} \frac{\Delta_{i_{1}} \cdots \Delta_{i_{l}}}{i_{1} ! \cdots i_{l} !}+R_{m+1},
\end{aligned}
$$

где для любого $x \in \mathbf{R}$

$$
\begin{aligned}
&\left|R_{m+1}\right| \leqslant \sum_{l=1}^{m} C_{n}^{l} \frac{B_{n-l}^{N(l)+m}(d)}{n^{N(l)+m}} \sum_{\substack{i_{1}, \ldots, i_{l} \geqslant 2 \\
i_{1}+\cdots+i_{l}=N(l)+m}} \frac{|\Delta|_{i_{1}} \cdots|\Delta|_{i_{l}}}{i_{1} ! \cdots i_{l} !} \\
&+ C_{n}^{m+1} \frac{|\Delta|_{2}^{m+1} B_{n-m-1}^{2(m+1)}(d / 2)}{(2 !)^{m+1} n^{2(m+1)}} \\
&+ \frac{2^{m+1}}{\pi} n C_{n}^{m+1} \alpha^{n-\nu-m-1}(T) \int_{T}^{\infty} \max ^{\nu}\left\{|f(t)|, \mathrm{e}^{-d|t|}\right\} d t \\
& T=\min \left\{\frac{1}{2|\Delta|_{2}}, \frac{1}{2 d}\right\} .
\end{aligned}
$$

Числа $N(l)$ можно выбрать следующим образом:

$$
\begin{aligned}
N(l) & =N\left(l ; n, m,|\Delta|_{2}, \ldots,|\Delta|_{m+2}\right) \\
& =\underset{l+1 \leqslant N \leqslant 2 l}{\arg \min }\left\{f(N): f(N)=C_{n}^{l} \frac{B_{n-l}^{N+m}}{n^{N+m}} \sum_{\substack{i_{1}, \ldots, i_{l} \geqslant 2 \\
i_{1}+\cdots+i_{l}=N+m}} \frac{|\Delta|_{i_{1}} \cdots|\Delta|_{i_{l}}}{i_{1} ! \cdots i_{l} !}\right\} .
\end{aligned}
$$

Здесь нам удобнее обозначить остаточную часть $R_{m+1}$, а не $R_{m+2}$, как в теореме 2. Разложение с индексами $N(l)=2 l$ естественно назвать длинным разложением. Подобные разложения выписаны во всех предыдущих утверждениях. Разло- 
жение с $N(l)=l+1$ короткое, остальные разложения средние. Разложение с индексами $N(l)$, определенными в (4), является разложением с наименьшей оценкой остаточной части из всех разложений в теореме 6.

4. Пояснения. Схема доказательства сформулированных утверждений следующая. Для нормированной $n$-кратной свертки распределения $P$ выписывается разложение Бергстрёма. Остаточная часть разложения Бергстрёма оценивается методом характеристических функций и, в случае разложений для распределений, с помощью неравенства Берри-Эссеена. Главные части разложения Бергстрёма раскладываются в ряды типа Эджворта, а для уменьшения оценки остаточных частей используется комбинаторное соотношение (лемма 1 ниже), которое позволяет оценить остаточную часть разложения, грубо говоря, следующими по порядку членами главного разложения и только ими (см. [8]).

Лемма 1. Пусть $F_{k}=\sum_{i=k}^{\infty} f_{i}, k \in \mathbf{N},-$ сходящиеся ряды вещественных чисел. Тогда для $F_{k}^{n}=\left(\sum_{i=k}^{\infty} f_{i}\right)^{n}$ справедливо соотношение

$$
F_{k}^{n}=\sum_{i=n k}^{m} \sum_{\substack{m_{0}+m_{1}+\cdots+m_{l}=n, m_{0} k+m_{1}(k+1)+\cdots+m_{l}(k+l)=i}} \frac{n !}{m_{0} ! m_{1} ! \cdots m_{l} !} f_{k}^{m_{0}} f_{k+1}^{m_{1}} \cdots f_{k+l}^{m_{l}}+R_{m+1}^{n},
$$

гde

$$
\begin{aligned}
R_{m+1}^{n}= & \sum_{\substack{m_{0}+m_{1}+\cdots+m_{l}=n, m_{0} k+m_{1}(k+1)+\cdots+m_{l}(k+l)=m+1, m_{l} \neq 0}} C_{m_{0}+\cdots+m_{l}}^{m_{1}+\cdots+m_{l}} f_{k}^{m_{0}} \cdots C_{m_{l-2}+\cdots+m_{l}}^{m_{l-1}+m_{l}} f_{k+l-2}^{m_{l-2}} \\
& \times\left(\sum_{i=0}^{m_{l-1}} C_{m_{l-1}+m_{l}-i-1}^{m_{l}-1} F_{k+l-1}^{i} f_{k+l-1}^{m_{l-1}-i}\right) C_{m_{l}}^{0} F_{k+l}^{m_{l}}
\end{aligned}
$$

(здесь $\left.n, m \in \mathbf{N}, l, m_{0}, \ldots, m_{l} \in \mathbf{Z}_{+}, n k \leqslant m\right)$.

3 а м е ч а н и е 5 . В (5) слагаемые $\left(n ! /\left(m_{0} ! m_{1} ! \cdots m_{l} !\right)\right) f_{k}^{m_{0}} f_{k+1}^{m_{1}} \cdots f_{k+l}^{m_{l}}$ можно заменить на $C_{m_{0}+\cdots+m_{l}}^{m_{1}+\cdots+m_{l}} f_{k}^{m_{0}} C_{m_{1}+\cdots+m_{l}}^{m_{2}+\cdots+m_{l}} f_{k+1}^{m_{1}} \cdots C_{m_{l-1}+m_{l}}^{m_{l}} f_{k+l-1}^{m_{l}-1} C_{m_{l}}^{0} f_{k+l}^{m_{l}}$. $\quad$ Величина $R_{m+1}^{n}$ зависит и от $k$.

Например, $\left(f_{1}+f_{2}+\cdots\right)^{3}=\left(f_{1}+F_{2}\right)^{3}=f_{1}^{3}+f_{1}^{2} F_{2}+f_{1} F_{1} F_{2}+F_{1}^{2} F_{2}$.

Автор благодарит В. В. Сенатова за обсуждение результатов работы.

\section{СПИСОК ЛИТЕРАТУРЫ}

1. Золотарев В. М. Аналог асимптотического разложения Эджворта-Крамера для случая сближения с устойчивыми законами распределения. - Труды VI Всесоюзного совещания по теории вероятностей и математической статистике. Вильнюс: Гос. изд-во полит. и науч. лит. ЛитССР, 1962, с. 49-50.

2. Золотарев В. М. Одномерные устойчивые распределения. М.: Наука, 1983, 304 с.

3. Ибрагимов И. А., Линник Ю. В. Независимые и стационарно связанные величины. М.: Наука, 1965, 524 с.

4. Осмоловский И. Ю. О некоторых свойствах многомерных аналогов многочленов Чебышёва-Эрмита. - Теория вероятн. и ее примен., 2008, т. 53, в. 2, с. 373-378.

5. Прохоров Ю. В., Розанов Ю. А. Теория вероятностей. М.: Наука, 1967, 495 с.

6. Сенатов В. В. Об одном многомерном аналоге разложения Чебышёва. - Теория вероятн. и ее примен., 2007, т. 52 , в. 3 , с. 603-610.

7. Сенатов B. В. Центральная предельная теорема: точность аппроксимации и асимптотические разложения. М.: Либроком, 2009, 350 с.

8. Сюлюкин A. В. Об асимптотических разложениях Бергстрёма-Чебышёва. - Теория вероятн. и ее примен., 2009, т. 54, в. 1, с. 176-185. 
9. Ярославчева Л. С. Неклассические оценки точности приближения асимптотическими разложениями в центральной предельной теореме. - Теория вероятн. и ее примен., 2008, т. 53, в. 2, с. 390-393.

10. Кристо $Г$. Асимптотические разложения в случае устойчивого предельного закона. I. - Литов. матем. сб., 1981, т. 21, № 2, с. 87-100.

11. Christoph G., Wolf W. Convergence Theorems with a Stable Limit Law. Berlin: Akademie Verlag, 1992, 200 p.

12. Миталаускас A., Статулявичус B. Асимптотическое разложение в случае устойчивого аппроксимирующего закона. - Литов. матем. сб., 1976, т. 16, № 4, с. 149166.

Поступила в редакцию

19.IV.2012

(c) 2013 г

\author{
JAIMUNGAL S. ${ }^{*}$, KREININ A.**, VALOV A.*** \\ THE GENERALIZED SHIRYAEV PROBLEM \\ AND SKOROKHOD EMBEDDING ${ }^{1)}$
}

Рассматривается связь между знаменитой проблемой вложения Скорохода и обратной задачей Ширяева для распределения момента первого достижения в случае броуновского движения: для заданного распределения вероятностей $F$ найти границу такую, что распределение момента первого ее достижения процессом есть $F$. Рандомизируя начальное состояние процесса, мы показываем, что обратная задача допускает аналитическое решение. Рандомизация начального состояния позволяет значительно расширить класс рассматриваемых распределений в случае линейной границы, а также выявить связь с проблемой вложения Скорохода.

Ключевые слова и фразы: момент первого достижения, обратная задача Ширяева о границе, вложение Скорохода.

1. Introduction. Let $X_{t}$ be an arbitrary process with the left-continuous sample paths and let $b(t)$ be a continuous absorbing boundary satisfying the condition $X_{0} \geqslant b(0)$. The random variable

$$
\tau= \begin{cases}\inf \left\{t \geqslant 0: X_{t}<b(t)\right\} & \text { if there exists } t \text { such that } X_{t}<b(t) \\ \infty & \text { otherwise, }\end{cases}
$$

is called the first hitting time for the process $X_{t}$. The problem

Given a process, $X_{t}$, and the boundary, $b(t)$,

find the distribution $F_{\tau}(t)=\mathbf{P}(\tau \leqslant t)$.

is the starting point of a very rich research area in the theory of stochastic processes. If $X_{t}$ is a diffusion process, the problem of finding the distribution of $\tau$ is a classical one. The first papers on the problem were published by P. Lévy, A. Khinchin, and A. Kolmogorov in the 1920s. These results are discussed in the monograph ${ }^{2)}$ by Khinchin [17]. Khinchin

* Department of Statistics, University of Toronto, Toronto, ON, Canada; e-mail: sebastian.jaimungal@utoronto.ca

** IBM Canada Ltd.; Department of Computer Sci., University of Toronto, Toronto, ON, Canada; e-mail: alex.kreinin@ca.ibm.com

*** Department of Statistics, University of Toronto, Toronto, ON, Canada; e-mail: valov@utoronto.ca

1) This research was supported in part by the Natural Sciences and Engineering Research Council (NSERC) of Canada.

${ }^{2)}$ Khinchin called (1) the second problem of diffusion. 